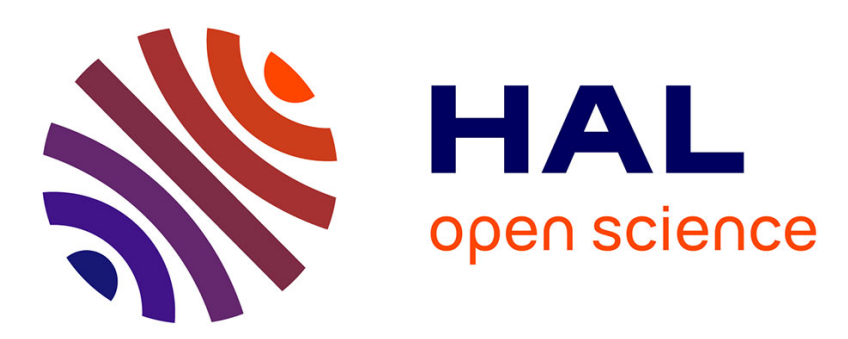

\title{
Dual Predictive Control of Electrically Stimulated Muscle using Biofeedback for Drop Foot Correction
}

Mitsuhiro Hayashibe, Qin Zhang, Christine Azevedo Coste

\section{To cite this version:}

Mitsuhiro Hayashibe, Qin Zhang, Christine Azevedo Coste. Dual Predictive Control of Electrically Stimulated Muscle using Biofeedback for Drop Foot Correction. IROS: Intelligent RObots and Systems, Sep 2011, San Francisco, United States. pp.1731-1736, 10.1109/IROS.2011.6094978 . lirmm00637760

\section{HAL Id: lirmm-00637760 \\ https://hal-lirmm.ccsd.cnrs.fr/lirmm-00637760}

Submitted on 2 Nov 2011

HAL is a multi-disciplinary open access archive for the deposit and dissemination of scientific research documents, whether they are published or not. The documents may come from teaching and research institutions in France or abroad, or from public or private research centers.
L'archive ouverte pluridisciplinaire HAL, est destinée au dépôt et à la diffusion de documents scientifiques de niveau recherche, publiés ou non, émanant des établissements d'enseignement et de recherche français ou étrangers, des laboratoires publics ou privés. 


\title{
Dual Predictive Control of Electrically Stimulated Muscle using Biofeedback for Drop Foot Correction
}

\author{
Mitsuhiro Hayashibe, Qin Zhang and Christine Azevedo-Coste
}

\begin{abstract}
Electrical stimulation (ES) is one of the solutions for drop foot correction. Conventional ES systems deliver predefined stimulation pattern to the affected muscles. However, timevariant muscle response may influence the gait performance as they are difficult to be taken into account in advance. Therefore, closed-loop ES control is important to obtain desired gait in presence of muscle response variation. In this work, a dual predictive control, which consists of two nonlinear generalized predictive controllers, is proposed to track desired torque. The stimulated muscle dynamics are modeled by Hammerstein cascades, with one representing stimulation to activation, the other representing activation to torque. Ankle dorsiflexion torque and ES-evoked EMG of tibialis anterior were recorded experimentally for model identification. The control scheme is validated by following desired torque trajectories with the identified model. The results show that the stimulation pattern obtained from the dual predictive control can produce good torque tracking according to the current muscle condition.
\end{abstract}

\section{INTRODUCTION}

Drop foot is a condition where an individual is not able to adequately dorsiflex or lift the foot. It is associated with a variety of conditions such as stroke, spinal cord injury (SCI), or cerebral palsy [1]. Regardless of the mechanism of injury, the drop foot condition can be improved by different techniques, which is typically referred to as drop foot correction. The main goal of drop foot correction is to provide toe clearance while the affected limb is swinging, and stability while the affected foot is on the ground, so as to prevent the dragging of the toe on the ground and decrease the risk of falls. Electrical stimulation (ES) is one of the existing solutions, which can artificially generate action potential in the place of central nervous system (CNS) for inducing muscle contraction. It was put forward for gait improvement since 1961 [2]. This technology presents its benefit compared to conventional ankle foot orthosis devices, in terms of the similar ability of gait improvement, the lightweight and better cosmesis, a decrease in spasticity, and an increase in walking speed [3].

The technology of ES for drop foot correction has advanced substantially over 40 years. A detailed review on this topic can be found in [1]. In such context, most interests were laid on designing stimulation system, aiming for optimal patterns and exploring gait sensors to detect gait events. In particular, optimal stimulation envelope is considered to be important to improve gait performance. The stimulation pattern commonly used in previous research is trapezoidal

M. Hayashibe, Q. Zhang, C. Azevedo-Coste are with DEMAR team, INRIA Sophia Antipolis, and LIRMM, UMR 5506 CNRS UM2, 161 Rue Ada, 34095 Montpellier Cedex 5, France. \{hayashibe, qin.zhang, azevedo\}@lirmm.fr
ES profile. However, it was reported that the muscle activity elicited by trapezoidal profile did not match the muscle activity during gait in able-bodied subject [4]. In addition, the muscle behavior generated by predefined stimulation may be influenced by time-variant muscle response such as muscle fatigue and spasticity. Therefore, it is important to adaptively modulate ES pattern in closed loop corresponding to the response variation. A closed-loop ES system, which is controlled by position sensors and triggered by footswitches with real time feedback, was proposed in [5]. The stimulation pattern can be adjusted through the information of the sensors. A micro-controller dynamically adjusting the ES intensity through a built-in algorithm was presented in [6]. The focus of this paper is the development of an novel ES control strategy to adaptively generate stimulation patterns for drop foot correction using biofeedback aiming for torque control rather than position control.

ES-evoked electromyography (eEMG) has been widely used to observe muscle electrical behavior such as [4] [7] as it permits noninvasive and reliable measurement of muscle activity. In [7], it was reported that the eEMG signal could be used for ES-induced torque prediction. Moreover, the mechanical response of stimulated muscle occurs later than the electrical response, due to so-called electromechanical delay (EMD). eEMG contains muscle contraction state prior to torque production. Thus it is advantage to use eEMG signal for feedforward control of muscle torque. In our previous work, a time-varying relationship during eEMG and ES-induced torque was reported in [8]. The follow-up study proposed to apply Kalman filter with forgetting factor to predict for muscle fatigue tracking based on eEMG [9]. Even when torque measurement is not available for certain time, it can contribute to torque prediction with biofeedback and the performance was validated in five SCI subjects [10]. In ES, it is important to realize smooth muscle activation transition due to the limitation of physiological response. Therefore, we were motivated to adopt a predictive control strategy based on identified muscle model which takes advantage of actual eEMG signal reflecting the actual muscle activation. Some simulation studies can be found in [12] and [13] to introduce predictive control strategy in different applications of ES. This work aims at proposing a closed-loop control strategy which can track desired torque trajectory rather than position one for drop foot correction. A dual predictive controller, that is, two nonlinear generalized predictive controllers in series to bridge the muscle input and output with intermediate variable from eEMG, is proposed in this paper. 


\section{Model Structure AND ITS ONLINE IDENTIFICATION}

\section{A. Model Structure}

The stimulation signal, the torque measurement, and the eEMG measurements can be used to identify the parameters of muscle models in muscle torque production as shown in Fig. 1. Excitation dynamics model, relating the stimulation $u_{s}(t)$ to the eEMG $y_{m}(t)$ (stim-to-eEMG), and contraction dynamics model, relating the eEMG $u_{m}(t)$ to the torque $y_{t}(t)$ (eEMG-to-torque) are considered as proposed in [7].

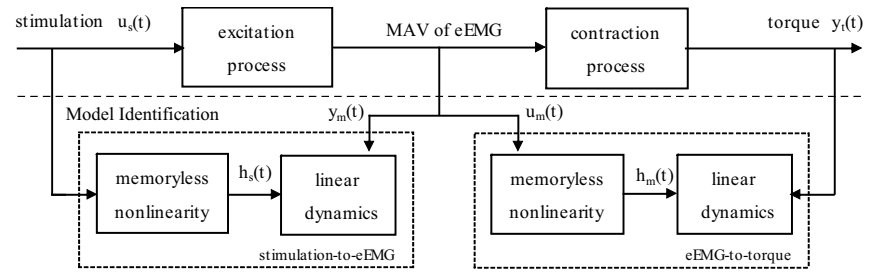

Fig. 1. Model structure of stimulated muscle for identification. The contraction dynamics model, relating eEMG to torque, the excitation dynamics model, relating stimulation to eEMG.

The discrete-time Hammerstein structure was used to model both excitation and contraction dynamics. For simplification, a generic Hammerstein structure was introduced here. It consists of a dynamic linear part and a memoryless nonlinear part. It is popularly used to represent highly nonlinear systems, such as electrically-stimulated muscles, relating stimulation to muscle force under isometric conditions [14].

In this work, the linear part is chosen as an autoregressive model with external input (ARX), which has been experimentally shown to yield good prediction of output force/torque in isometric situation [15].

$$
A\left(z^{-1}\right) y(t)=B\left(z^{-1}\right) h(t)
$$

in which $A, B$ are defined as

$$
\begin{aligned}
& A\left(z^{-1}\right)=1+a_{1} z^{-1}+a_{2} z^{-2}+\cdots+a_{l} z^{-l} \\
& B\left(z^{-1}\right)=b_{1} z^{-1}+b_{2} z^{-2}+\cdots+b_{m} z^{-m}
\end{aligned}
$$

where $z^{-1}$ is the backward shift operator. In (1), $y(t)$ is the output of the linear part, the intermediate variable $h(t)$ is the input of the linear part, as well as the output of the nonlinear part with following polynomial formulation:

$$
h(t)=\sum_{i=0}^{n} \gamma_{i} u^{i}(t)
$$

Substituting (2), (3) into (1), the output of a polynomial Hammerstein model (PHM) at a given time $t$ can be parameterized as:

$$
y(t, \boldsymbol{\theta})=\sum_{i=1}^{l} a_{i} y(t-i)+\sum_{i=1}^{m} \sum_{j=0}^{n} b_{i} \gamma_{j}(u(t-i))^{j}
$$

where $\boldsymbol{\theta}=\left[a_{1}, \cdots, a_{l}, b_{1}, \cdots, b_{m}, \gamma_{0}, \cdots, \gamma_{n}\right]^{T}$ is a parameter vector containing the model parameters. Therefore, there are $l+m+n+1$ model parameters required to be identified in a PHM $(1, m, n)$ model.

\section{B. Identification of Time-varying Parameters}

The state-space form is basically required for the implementation of Kalman filter. Considering a PHM $(1, \mathrm{~m}, \mathrm{n})$, its state-space form can be compactly written as:

1) process equation

$$
\mathbf{x}_{k}=\mathbf{A} \mathbf{x}_{k-1}+\mathbf{B} \sum_{i=0}^{n} \gamma_{i}\left(u_{k-1}\right)^{i}
$$

2) measurement equation

$$
y_{k}=\mathbf{C x}_{k}
$$

where $u_{k-1}$ is the previous model input. Subscript $k$ denotes the current time step. The current state vector can be described as $\mathbf{x}_{k}=\left[x_{1, k}, x_{2, k}, \cdots, x_{q, k}\right]^{T}$, where $q=\max \{l, m\}$. $\mathbf{A} \in \mathbb{R}^{q \times q}$ correlates the previous states to the current states. $\mathbf{B} \in \mathbb{R}^{q \times 1}$ correlates the previous inputs to the current states. They can be represented as follows:

$$
\mathbf{A}=\left[\begin{array}{cccccc}
a_{1} & 1 & 0 & \cdots & 0 & 0 \\
a_{2} & 0 & 1 & \cdots & 0 & 0 \\
\vdots & \vdots & \vdots & \ddots & \vdots & \vdots \\
a_{q-1} & 0 & 0 & \cdots & 0 & 1 \\
a_{q} & 0 & 0 & \cdots & 0 & 0
\end{array}\right], \quad \mathbf{B}=\left[\begin{array}{c}
b_{1} \\
b_{2} \\
\vdots \\
b_{q-1} \\
b_{q}
\end{array}\right]
$$

$\mathbf{C} \in \mathbb{R}^{1 \times q}$ correlates the current states to the current measurement with the following expression:

$$
\mathbf{C}=\left[\begin{array}{lllll}
1 & 0 & \cdots & 0 & 0
\end{array}\right]
$$

The detailed implementation of Kalman filter can be found in [10]. At each time step, the internal states and model parameters were simultaneously identified by Kalman filter and measurement update. Notice that, the parameters relating to linear part $b_{i}(i=1, \cdots, q)$ and nonlinear part $\gamma_{j},(j=0, \cdots, n)$ in (4) were separately identified, rather than identifying their products as in [9]. This improvement is meaningful for directly applying predictive control scheme on the linear part of a PHM model.

\section{CONTROLler DESIGN}

\section{A. Dual Predictive control}

We propose a dual predictive controller which consists of two nonlinear generalized predictive controllers in series in order to track desired torque during electrical stimulation. Our main idea is to use eEMG signal for a dual-purpose in this control strategy, eEMG has some properties of capturing all of neural excitation, directly from stimulation, and also from muscle fatigue, reflex and spasticity [7]. Fig. 2 demonstrates the control strategy we propose. There are two controllers in series to handle predictive control of stimulated muscle enhanced by biofeedback. Activation controller takes eEMG as control signal $m_{d}$ to drive the predicted torque $y_{p}$, close to the desired torque trajectory $y_{d}$, based on contraction dynamics model. Stimulation controller uses $m_{d}$ obtained from activation controller as the desired eEMG trajectory, so that the control signal, stimulation pulse width $u_{s}$, can be computed to drive predicted eEMG $m_{p}$ close to $m_{d}$, based on excitation dynamics model. 


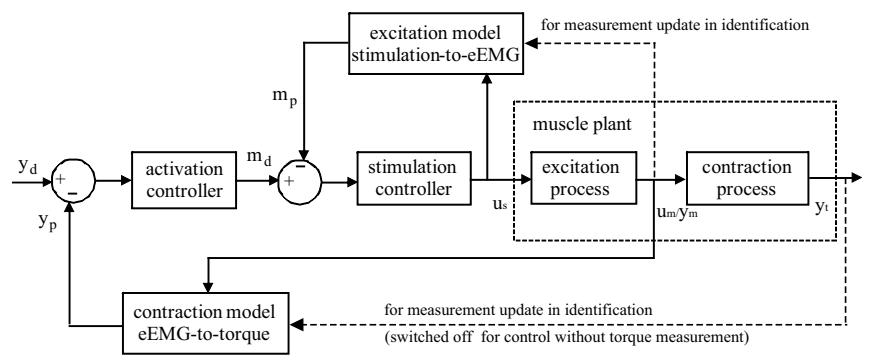

Fig. 2. Diagram illustrating the dual predictive control using biofeedback. The control signal obtained from activation controller is used as desired reference of stimulation controller. In each controller, nonlinear generalized predictive control algorithm was applied based on a PHM model. The torque measurement $y_{t}$ and eEMG measurement $u_{m} / y_{m}$ were only used for model identification as shown in dashed lines.

In both the activation controller and the stimulation controller, the same model structure - the polynomial Hammerstein model - is used for precess prediction and optimization. Therefore, the overall control problem can be reduced to resolve a nonlinear generalized predictive control problem in which a polynomial Hammerstein model is utilized to represent the dynamics process.

\section{B. Nonlinear Generalized Predictive Control}

As a whole, the solution of a nonlinear generalized predictive controller consists of two parts: linear part and nonlinear part. The control solution of the linear part is first considered by generalized predictive control (GPC) algorithm, which has been described in several publications [11] [16] [17]. Despite different methods can be used to obtain the control law of GPC, the general idea is to minimize a multistage cost function given by

$$
J=\sum_{j=1}^{N_{p}} \xi_{j}\left[\hat{y}_{k+j \mid k}-v_{k+j}\right]^{2}+\sum_{j=1}^{N_{u}} \delta_{j}\left[\Delta h_{k+j-1}\right]^{2}
$$

Where $\hat{y}_{k+j \mid k}$ is an optimum $j$-step ahead prediction of the controlled variable using data up to time instant $k, v_{k+j}$ is the future reference trajectory, $\Delta h_{k+j-1}$ is the increment of manipulated variable. Weighting coefficients $\xi_{j}, \delta_{j}$ respectively penalize relative big changes of $\hat{y}_{k+j \mid k}$ and $\Delta h_{k+j-1}$. $N_{p}$ is known as prediction horizon, and control horizon $N_{u}$, $1 \leq N_{u} \leq N_{p}$, implies that all the increments of the control effort are assumed to be zero for $j>N_{u}$.

A simpler formulation of GPC can be found in [18], which was introduced instead of solving recursive Diophantine equations. In a word, the optimization problem can be computed online and in real time in terms of the manipulated variable sequences $\left[h_{k \mid k}, h_{k+1 \mid k}, \cdots, h_{k+N_{u}-1 \mid k}\right]$, so that the predicted controlled variables $\left[\hat{y}_{k+1 \mid k}, \hat{y}_{k+2 \mid k}, \cdots, \hat{y}_{k+N_{p} \mid k}\right]$ follow a desired reference trajectory $\left[v_{k+1}, v_{k+2}, \cdots, v_{k+N_{p}}\right]$. Only the first element of the control sequence is actually implemented during time interval $[k, k+1]$, that is, $h_{k}=h_{k \mid k}$. Then the procedure is repeated at the next sampling time.

In this way, GPC has four tuning parameters: $N_{p}, N_{u}$, $\xi$ and $\delta$. Usually, the selection of prediction horizon $N_{p}$ relies on sampling time. The selection of control horizon
$N_{u}$ depends on a trade-off between reducing computation amount and achieving global optimization [19]. A large $N_{u}$ may avoid constraints violation before they are arrived at, but may result in substantial amount of computation due to involved inversion of matrix [11]. The effect of $\delta$ relates on suppressing aggressive control action, while $\xi$ allows to assign weight to reduce the prediction error for trajectory tracking.

The control signal $h_{k}$ obtained by GPC is a solution of the linear predictive control problem at step $\mathrm{k}$, which is required to be applied to the linear part of the system. It is also provided to generate the plant input $u_{k}$ on the basis of function (3). The nonlinear problem can be stated that, at each time step, the signal $h_{k}$ is obtained as described above, the nonlinear model coefficients $\gamma_{0}, \cdots, \gamma_{n}$ are known by model identification, we need to find the control input signal $u_{k}$. It can be considered as finding zeros of the following function

$$
p\left(u_{k}\right)=\gamma_{0}+\gamma_{1} u_{k}+\gamma_{2} u_{k}^{2}+\cdots+\gamma_{n} u_{k}^{n}-h_{k} .
$$

In this work, the $u_{k}$ was calculated by finding eigenvalues using Frobenius companion matrix. Until now, the control problem of a nonlinear generalized predictive controller is solved in two steps, first linear solution, and then nonlinear solution.

\section{Closed-Loop Implementation of the Dual Predictive Con- troller}

Both the activation controller and the stimulation controller include the procedure described in III. B. In the dual predictive controller we proposed in Fig. 2, the control signal obtained from activation controller was treated as desired reference of stimulation controller. The closed-loop implementation of the dual predictive controller consists of the following events periodically:

1) Obtain stimulation signal, eEMG, and output torque (at time instant $\mathrm{t}$ )

2) Update the model parameter estimates by Kalman filter for both muscle excitation model and contraction model. Note that, both the linear part parameters $a_{i}$, $b_{i}$ and the nonlinear part parameters $\gamma_{j}$ in (4) are simultaneously identified

3) Using linear part of the identified contraction model, the intermediate signal $h_{m}(t)$ is computed by GPC

4) The control signal of activation controller $m_{d}$ is calculated using (8), and then is considered as desired reference of stimulation controller

5) Using linear part of the identified excitation model, the intermediate signal $h_{s}(t)$ is computed by GPC

6) The control signal of stimulation controller $u_{s}$ is calculated using (8)

7) Apply $u_{s}$ to the muscle

8) Wait for the next sample then go to 1). 


\section{RESUlts}

\section{A. Experiment for Model Identification}

In order to get a realistic model for our simulation study, we used experimental data to identify model parameters. Two healthy subjects participated in this study. The experimental set-up is depicted in Fig. 3. The subjects were seated on a chair with the right ankle at $90^{\circ}$, while the foot was strapped on a pedal. A classical stimulation scheme was used to induce ankle dorsiflexion. The active (cathode) stimulating electrode was placed over the common peroneal nerve and the indifferent (anode) was placed over the tibialis anterior (TA) muscle. Sequences of $2 \mathrm{~s}$ stimulation and $2 \mathrm{~s}$ rest were applied for 30 minutes. A trapezoidal envelope with pulse width modulation was applied. Each $2 \mathrm{~s}$ stimulation train consists of $0.4 \mathrm{~s}$ ramp-up, $1.2 \mathrm{~s}$ plateau and $0.4 \mathrm{~s}$ ramp-down. The common peroneal nerve was stimulated with pulse width modulation where the maximum pulse width $\left(P W_{\max }\right)$ was fixed at $350 \mu \mathrm{s}$, constant stimulation frequency at $40 \mathrm{~Hz}$, under isometric conditions by a programmable stimulator (ProStim, MXM, France). The constant stimulation amplitude was varied from 30 to $46 \mathrm{~mA}$ depending on the subjects.

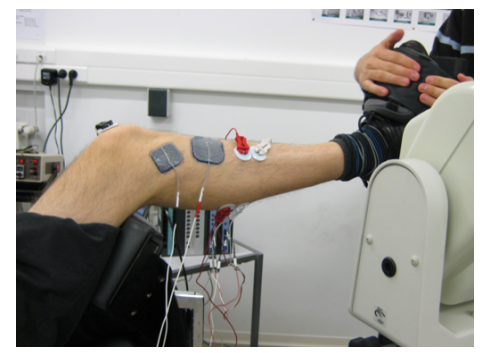

Fig. 3. Experimental set-up for stimulation and ankle torque measurement.

Evoked EMG activity of TA was recorded, amplified (gain 1,000) and sampled at $4 \mathrm{kHz}$ by an acquisition system (Biopac MP100, Biopac Systems Inc., CA, USA). Two surface electrodes were positioned over the TA muscle in the direction of muscle fiber with $20 \mathrm{~mm}$ interelectrode spacing. The reference electrode was placed on the patella. The skin under the electrodes was shaved to minimize the impedance. Isometric ankle dorsiflexion torque was measured using a calibrated dynamometer (Biodex 3, Shirley corp., NY, USA) at $2 \mathrm{kHz}$, interfaced with the acquisition system.

\section{B. Data Processing}

The stimulation artifacts were removed by means of blanking window to extract muscle response (Mwave). The measured torque was offset with respect to the baseline of the torque measurement without stimulation. The eEMG data was then divided into epochs with each epoch containing one Mwave. The mean absolute value (MAV) of eEMG and mean torque during the epoch was simultaneously calculated and normalized by their maximum values. In this work, the stimulation pulse width, MAV of eEMG and mean torque are used to identify both muscle excitation model and muscle contraction model. Fig. 4 shows an example of the processed result.
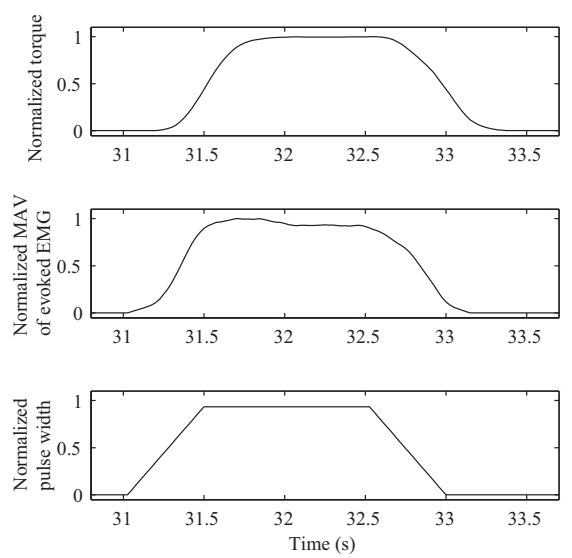

Fig. 4. An example of processed data. Normalized torque, normalized MAV of eEMG and normalized stimulation pulse width were prepared for model identification. The muscle mechanical response (torque) occurs later than muscle electrical response (eEMG) due to so-called electromechanical delay (EMD). This phenomenon is one of the advantages of using eEMG feedback for predictive torque control.

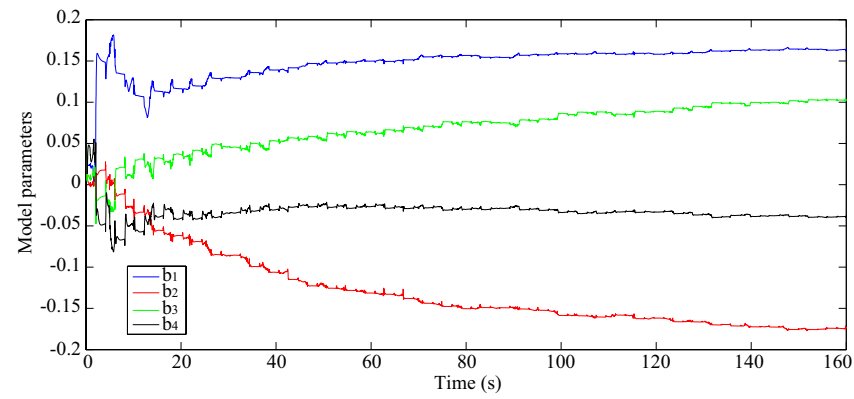

Fig. 5. Model parameters of the linear part of the input in contraction model in subject2. The parameters were estimated by Kalman filter and represent global time-varying property.

\section{Identification Result}

The parameters of muscle excitation and contraction model were identified by Kalman filter with forgetting factor. The parameters of muscle excitation model were identified based on stimulation pulse width and MAV of eEMG. The parameters of muscle contraction model were identified based on MAV of eEMG and torque. For each model, the parameters relating to linear and nonlinear parts were separately identified as mentioned before. Model order $l=$ $3, m=4, n=3$ was chosen for both excitation and contraction models as proposed in previous work [9]. All the initial states were fixed at zeros. The linear parameters $a_{i}$ were initialized at 0.2 , and $b_{i}$ at 0.1 . The nonlinear parameters $\gamma_{j}$ were initialized at $0.0,1.0,1.0,1.0$. In the presented results, forgetting factor was selected at 0.999 . Fig. 5 demonstrates a part of parameters of contraction model in subject2. It reveals that, the parameters represent global time-varying property during the electrical stimulation.

\section{Simulation Study of Control Strategy}

In order to investigate the performance of the proposed dual predictive controller described in section III. A, the closed-loop simulations were carried out while the muscle 
model was identified using real experimental data to simulate real muscle response.

The relevant tuning parameters were chosen as below. The sampling time was set at $0.025 \mathrm{~s}$. The prediction horizon $N_{p}$ and control horizon $N_{u}$ were set at $N_{p}=30, N_{u}=20$. The control input $u$ was constrained in $[0,1]$ representing respectively non fiber recruitment and maximal fiber recruitment. For convenience, the weighting coefficients of controlled variables $\xi_{1}$ (in activation controller) and $\xi_{2}$ (in stimulation controller) were fixed as $\xi_{1}=\xi_{2}=1$, while the weighting coefficients of control signals $\delta_{1}$ and $\delta_{2}$ were constant and adjustable. Finally, the tuning problem was simplified to a tuning of parameters $\delta_{1}$ and $\delta_{2}$.

The control task of this work is calculating appropriate and corrective stimulation signal in order to track the desired ankle torque trajectory. In order to assess the suitability of the proposed dual predictive controller, the effects of stimulation constraints, the stability of the stimulation, and the control performance were evaluated as follows.

Effects of Control Constraints: The desired torque reference consists of a sequence of two $4 \mathrm{~s}$ trains, where the first $2 \mathrm{~s}$ were increased from a fixed minimum value to a predetermined maximum value, and then the next $2 \mathrm{~s}$ were symmetrically decreased. The maximum value was chosen to be $2 \mathrm{Nm}$ and $4 \mathrm{Nm}$, respectively. The simulation result is shown in Fig. $6\left(\delta_{1}=0.5, \delta_{2}=5\right)$. The proposed dual predictive controller was used to generate the stimulation pulse width to drive the predicted torque as close as possible to the desired torque. However, when the desired torque is above $3.4 \mathrm{Nm}$, the stimulation pulse width represents saturation, which is important to avoid any damage of the patients' muscle due to excessive stimulation. It could be performed with the constraints condition in predictive control.

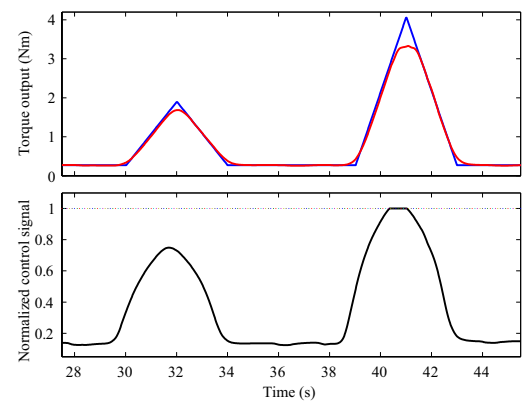

Fig. 6. Torque reference (blue) and reproduced torque (red). The control input was constrained in $[0,1]$ to guarantee stimulation safety of the patient.

Torque Tracking performance: This desired torque trajectory consists of a sequence with two trains. One train is with $4.5 \mathrm{~s}$ square profile, where the maximum value is fixed at $2.4 \mathrm{Nm}$. Another train is with $6.5 \mathrm{~s}$ trapezoidal profile, where the maximum value is fixed at $2.4 \mathrm{Nm}$ and lasts 2.5s. Fig. 7 shows the tracking performance to the desired torque trajectory. Three sets of weighting coefficients were tested. The weighting coefficients of input in two controllers were respectively referred to as DPC1 $\left(\delta_{1}=0.1, \delta_{2}=20\right)$, $\operatorname{DPC} 2\left(\delta_{1}=0.5, \delta_{2}=5\right)$ and DPC3 $\left(\delta_{1}=20, \delta_{2}=2\right)$. We can find that, on one hand, different weighting coefficients lead to different time converging to the desired torque, on the other hand, even different weighting coefficients were selected, the output torque can track the torque reference in a limited time. The transient processes are smooth in both torque tracking and control input, which is important for muscle to gradually respond to the stimulation within the physiologically feasible velocity. It also matches the intuitive requirement of muscle response during electrical stimulation. In particular, comparing these two different torque types, the ramp-up period in trapezoidal is important to reduce spasticity and fatigue due to fast contraction, and the rampdown period is important to avoid foot-flap or foot-slap [1], thus the trapezoidal profile is more practical than square profile in practice. The tracking performance of trapezoidal torque is better than the tracking of square torque. That implies that a realistic torque trajectory can ensure better controller performance. Moreover, even if an unrealistic torque trajectory is designed by mistake, the controller can generate more practical stimulation signal by adjusting the weighting coefficients as DPC2 in Fig. 7. This feature is important since there is a limitation of muscle reactive velocity, the proposed controller has an ability to explicitly ensure practical stimulation transition. Finally, weighting factors $\left(\delta_{1}=0.5, \delta_{2}=5\right)$ were considered as an optimal choice. They were used in the following study.
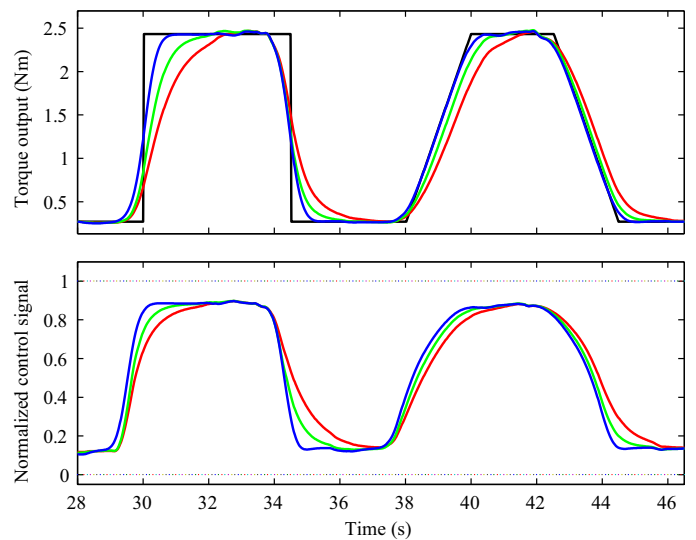

Fig. 7. Comparison of tracking performance with different types of torque trajectory. Different weighting coefficients were also tested. The weighting coefficient of input was chosen as DPC1 (red, $\left.\delta_{1}=0.1, \delta_{2}=20\right)$, DPC2 (green, $\delta_{1}=0.5, \delta_{2}=5$ ) and DPC3 (blue, $\delta_{1}=20, \delta_{2}=2$ ).

Robustness of the Dual Predictive Controller: In order to assess the control performance with the proposed method, we assumed that torque measurement was interrupted after certain time. The updates of model parameters were switched off after 19.2s for both the excitation and contraction model. Consequently, the model prediction in the control after 19.2s was only driven by the model input and the model parameter estimates at the time of 19.2s. The dual predictive controller can still generate suitable control signal to obtain desired torque trajectory as shown in Fig. 8. That is quite useful for ES control in the presence of sensor failure or control only based on biofeedback. The control performance was 

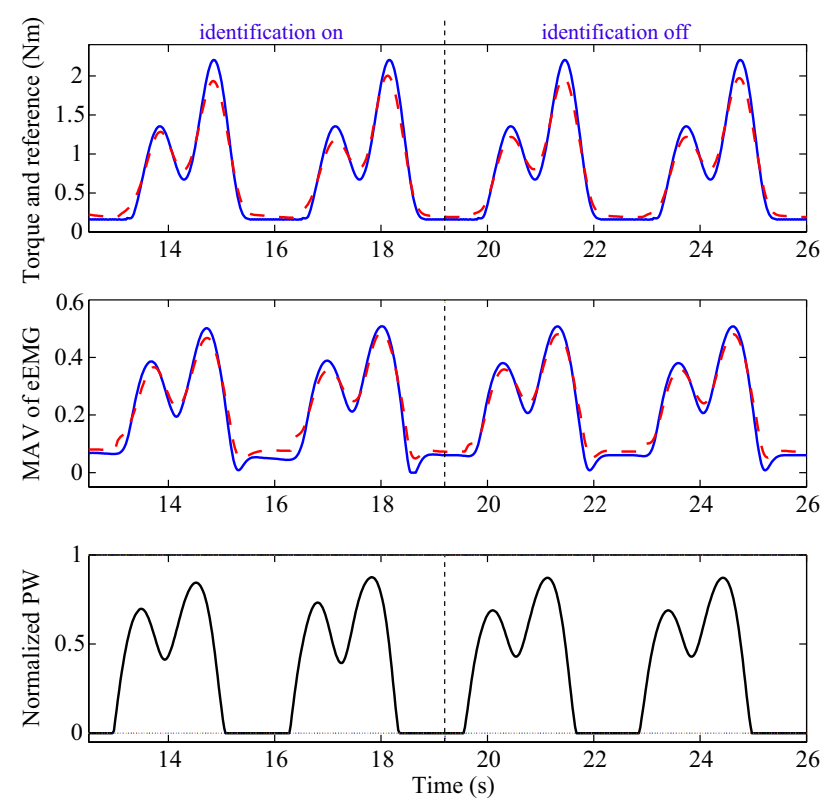

Fig. 8. Torque reference (solid blue) and reproduced torque output by the proposed controller (dashed red). The updates of model parameters were switched off after 19.2s. Consequently, the model prediction in the control was only driven by the model input and the model parameter estimates at the time of $19.2 \mathrm{~s}$.

maintained between before and after switching off model identification. It shows that the stability of the controller even in the condition where the model is time-varying or not. The reference trajectory here was prepared according to the recommended profile as natural TA contraction during gait in [4]. Another advantage of the proposed framework is that any reference can be input and the stimulation patterns can be systematically generated with explicit consideration of muscle activity.

\section{CONCLusions}

In the context of drop foot correction through electrical stimulation, optimal stimulation profile is important to improve gait performance. However, traditional predefined stimulation profile may suffer from muscle time-variant property. Hence it is valuable to adaptively modulate stimulation pattern in order to obtain desired gait performance and avoid over-stimulation as well. In this work, we aim at the development of an ES control strategy for drop foot correction which allows torque tracking. Classical ES strategy was conducted on two healthy individuals to induce dorsiflexion through surface electrodes. ES-evoked EMG signal and ankle torque were recorded for the model identification, which was performed by Kalman filter with forgetting factor. A dual predictive controller was proposed and verified by generating control signal adaptively according to the current muscle condition. The control signals were constrained to guarantee stimulation safety of the patient. The simulation results represent good torque tracking performance, effective safety and systematic input pattern generation. The muscle response against stimulation is quite different depending on the patients. This method can contribute to a generation of stimulation considering the actual muscle activation state. The future work will be extended to real time implementation on hemiplegic patients as well as dynamic motion tracking.

\section{REFERENCES}

[1] G. M. Lyons, T. Sinkjær, J. H. Burridge, and D. J. Wilcox, "A Review of Portable FES-Based Neural Orthoses for the Correction of Drop Foot", IEEE Transactions on Neural Systems and Rehabilitation Engineering, vol. 10, 2002, pp. 260-279.

[2] W. Liberson, H. Holmquest, and M. Scott, "Functional Electrotherapy: Stimulation of the Common Peroneal Nerve Synchronised with the Swing Phase of Gait of Hemiplegic Subjects", Archives of Physical Medicine and Rehabilitation, vol. 42, 1961, pp. 202-205.

[3] I. Swain, P. Taylor, "The Clinical Use of Functional Electrical Stimulation in Neurological Rehabilitation", Horizons in Medicine $16 \mathrm{C}$ Updates on major clinical advances, Royal College of Physicians, London, 2004, pp. 315-322.

[4] D. T. O'Keeffe, A. E. Donnelly and G. M. Lyons, "The Deveplopment of a Potential Optimized Stimulation Intensity Envelope for Drop Foot Applications", IEEE Transactions on Neural Systems and Rehabilitation Engineering, vol. 11, 2003, pp. 249-256.

[5] Y. L. Chen, Y. C. Li, T. S. Kuo and J. S. Lai, "The Development of a Closed-loop Controlled Functional Electrical Stimulation (FES) in Gait Training", Journal of Medical Engineering \& Technology, vol. 25, 2001, pp. 41-48.

[6] P. P. Breen, D. T. O'Keeffe, R. Conway, and G. M. Lyons, "A System for the Delivery of Programmable, Adaptive Stimulation Intensity Envelopes for Drop Foot Correction Applications", Medical Engineering \& Physics, vol. 28, 2006, pp. 177-186.

[7] A. Erfanian, H. J. Chizeck, and R. M. Hashemi, "Using Evoked EMG as a Synthetic Force Sensor of Isometric Electrically Stimulated Muscle", IEEE Tran. on Biomedical Engineering, vol. 45, 1998, pp. 188-202.

[8] Q. Zhang, M. Hayashibe, et al., "Torque Prediction Using Stimulus Evoked EMG and its Identification for Different Muscle Fatigue States in SCI Subjects", in Int. Conf. of IEEE Engineering in Medicine and Biology Society, Buenos Aires, Argentina, pp. 3523-3526, 2010.

[9] Q. Zhang, M. Hayashibe, D. Guiraud, "Muscle Fatigue Tracking based on Stimulus Evoked EMG and Adaptive Torque Prediction", in IEEE Int. Conf. on Robotics and Automation, Shanghai, China, pp. 10001016, 2011.

[10] Q. Zhang, M. Hayashibe, P. Fraisse, D. Guiraud, "FES-Induced Torque Prediction with Evoked EMG Sensing for Muscle Fatigue Tracking", IEEE/ASME Transactions on Mechatronics, (in print), 2011.

[11] E. F. Camacho and C. Bordons, Model Predictive Control, Springer, New York, 1999.

[12] S. Mohammed, P. Poignet, P. Fraisse and D. Guiraud, "Lower Limbs Movement Restoration using Input-Output Feedback Linearization and Model Predictive Control", in IEEE/RSJ Int. Conf. on Intelligent Robots and Systems, San Diego, CA, USA, 2007, pp. 1945-1950.

[13] R. M. Esfanjani, F. Towhidkhah, "Application of Nonlinear Model Predictive Controller for FES-Assisted Standing Up in Paraplegia”, in IEEE Engineering in Medicine and Biology 27th Annual Conference, Shanghai, China, 2005, pp. 6210-6213.

[14] T. L. Chia, P. Chow and H. J. Chizeck, "Recursive Parameter Identificaiton of Constrained Systems: An Applicaiton to Electrically Stimulated Muscle", IEEE Transaction on Biomedical Engineering, vol. 38, pp. 429-442, 1991.

[15] L. A. Bernotas, P. E. Crago, and H.J. Chizeck, "A Discrete-Time Model of Electrically Stimulated Muscle", IEEE Transaction on Biomedical Engineering, vol. BME-33, pp. 829-838, 1986.

[16] K. Warwick, D. W. Clarke, "Weighted Input Predictive Controller", IEEE proceedings, vol. 135, 1988, pp 16-20.

[17] Q. M. Zhu, K. Warwick and J. L. Douce, "Adaptive general predictive controller for nonlinear systems", IEEE proceedings-D, vol. 138, 1991, pp 33-40.

[18] P. Albertos and R. Ortega, "On Generalized Predictive Control: Two Alternative Formulations", Automatica, vol. 25, 1989, pp 753-755.

[19] M. A. Henson, "Nonlinear Model Predictive Control: Current Status and Future directions", Computers and Chemical Engineering, vol. 23, 1998, pp 187-202. 